East African Medical Journal Vol. 86 (Supplement) December 2009

CLINICO-PATHOLOGIC CHARACTERISTICS AND TREATMENT OUTCOMES OF CHILDREN WITH NEUROBLASTOMA AT THE KENYATTA NATIONAL HOSPITAL , NAIROBI

G. W. Kitonyi, MBChB, FRC Path, Senior Lecturer, Haematology and Blood Transfusion Unit, Department of Human Pathology, W. M. Macharia, MBChB, MMed (Nbi), MSc (McMaster), Dip. Haem-Oncol (MacMaster), Associate Professor, Department of Paediatrics and Child Health, O. W Mwanda, MBChB, FRC Path (Associate), MD, Associate Professor and R. Pamnani, MBBS, MD(Path), Lecturer, Haematology and Blood Transfusion Unit, Department of Human Pathology, College of Health Sciences, University of Nairobi, P. O. Box 19676 - 00202, Nairobi, Kenya

Request for reprints to: Dr. G. W. Kitonyi, P.O. Box 21494-00505, Nairobi, Kenya

\title{
CLINICO-PATHOLOGIC CHARACTERISTICS AND TREATMENT OUTCOMES IN CHILDREN WITH NEUROBLASTOMA AT THE KENYATTA NATIONAL HOSPITAL, NAIROBI
}

\author{
G. W. KITONYI, W. M. MACHARIA, O. W. MWANDA and R. PAMNANI
}

\begin{abstract}
Objective: To determine clinical-pathologic characteristics, treatment modalities and treatment outcomes of children diagnosed with neuroblastoma.

Design: Cross- sectional descriptive study based on secondary data from patient records.

Setting: Records department of Kenyatta National Hospital (KNH), a tertiary teaching and referral hospital based in Nairobi.

Subjects: Children aged 15 years and below, admitted with the diagnosis of neuroblastoma, between January 1997 and December 2005.

Main outcome measures: Presenting clinical features, diagnostic modalities including laboratory and imaging data, treatment modalities, response to treatment and patient survival.

Results: Twenty six patients were eligible for the study; 13 males and 13 females giving a M: F ratio of $1: 1$. The age range was 5 days to 12 years, with a median age of five years. Abdominal swelling (53.8\%), inability to walk due to bone pains, $(50 \%)$, and cranial or periorbital swelling, $(38.5 \%)$ were the commonest presenting features. Diagnosis of neuroblastoma was based on tissue biopsy in $50 \%$ (95\% CI 40.6-79.8\%) of the patients, and on fine needle aspiration cytology of mass or bone marrow in the rest. Bone marrow involvement was present in 16, (75\%). Anaemia, was common with $72.7 \%$ patients having a haemoglobin $(\mathrm{HB})<8 \mathrm{~g} / \mathrm{dl}$ at presentation. Immunohistochemistry and cytological grading were done in two, $(8 \%)$, patients. Urinary vanilly 1 mandelic acid (VMA), screening was positive in $50 \%$ (95\% CI 29.9\%$70.1 \%)$. The most frequently involved organs were abdomen $(88.9 \%)$, and skeleton, $(84.6 \%)$. Majority of patients, $(92.3 \%)$, presented with advanced stage IV disease. Three patients died before commencement of treatment. All treated patients $(100 \%)$, received cytotoxic therapy. Only two patients $(8.6 \%)$ had surgery as part of treatment while one, $(4.3 \%)$ was treated with radiotherapy. The initial treatment regimen was similar for all the patients. Although most patients had a complete initial response to treatment, early relapse, treatment failure, death or loss to follow up of patients with progressive disease were common. Overall survival (OS) at one year and two years were $19.2 \%(95 \% \mathrm{CI} 6.6-39.4 \%)$ and $7.7 \%$ (95\% CI $0.9 \%-251 \%)$ respectively. Only one patient was alive, (also free of disease), five years after diagnosis.

Conclusion: Although other clinical- pathologic findings of the patients were similar to those reported elsewhere, virtually all study patients presented with advanced stage IV disease, which would be associated with poor prognosis irrespective of quality of care. Priority must therefore be on ensuring early diagnosis and referral of patients with neuroblastoma before any other interventions can be expected to positively impact on outcome. The limited role of surgery and radiotherapy observed over the study period may be attributed to late presentation of the patients. Pathologic evaluation of patients was inadequate, to some extent due to unavailability of facilities, but extra
\end{abstract}




\begin{abstract}
important information could have been availed at minimal extra cost. To be at par with current internationally accepted treatment approaches that have been associated with improved survival, there is need to base choice of regimens for individual patients on clinical and readily accessible pathologic markers.
\end{abstract}

\section{INTRODUCTION}

Neuroblastoma, a malignant childhood embryonal tumour of the sympathetic neuroblasts is the most common extra- cranial solid childhood tumour in the developed world, making up $8 \%$ of all malignant neoplasms in children (1).The tumour arises from sites where these tissues are distributed and spreads by contiguous extension, haematogenous or lymphatic tissues spread. Common metastatic sites are the liver, bone marrow and skin. Although neuroblastoma is less common in African children, a significant number of children are diagnosed with neuroblastoma each year, leading to considerable morbidity and mortality. In a study involving 787 cases of childhood cancers at $\mathrm{KNH}$, (excluding retinoblastoma and brain tumours), Macharia(2) found a relative neuroblastoma frequency of $3.3 \%$.

Over half of neuroblastoma patients present before or at the age of twoyears. Rarely, neuroblastoma maybe congenital. Patients commonly present with abdominal swelling, orbital and periobital masses, manifesting as proptosis, exophthalmos, and periorbital oedema. Bone and joint pains, walking difficulties, and inability to stand, occur due to skeletal infiltration. Bone marrow involvement is present in over half of all patients at presentation, leading to anaemia and occasional thrombocytopenia. Neurological symptoms may arise from cord compression or cranial extension of the tumour. A proportion of patients present with chest tumours, at times giving rise to superior vena caval syndrome. Rarely, Horner's syndromemay be encountered when there is neck involvement. In neonates subcutaneous nodules are sometimes present. Non specific clinical features include irritability, lassitude, weight loss, fever, vomiting and diarrhoea. Symptoms associated with catecholamine production and vasoactive intestinal polypeptide syndrome maybe present in occasional patients (1).

Ideally all neuroblastoma patients should have tissue biopsy and histopathology diagnosis, which should include morphological grading of the tumour as either high risk, (associated with poor prognosis), or low risk (3). Neuroblastomas are generally small round blue cell tumours which sometimes exhibit pseudorossete formation. In histology and cytology materials, some neuroblastomas may be indistinguishable from other small round blue cell tumours. In these difficult cases immunohistochemistry (4) and measurement of catecholamine metabolites vanillymandelic acid (VMA) or homovanilylmadelic acid (HVMA), are useful in arriving at a firm diagnosis. VMA and HVA have been reported as positive in up to $90 \%$ of patients with highly sensitive chromatographic tests (5). Measurement of VMA is helpful in resource limited set ups whereimmunohistochemistry maybe prohibitively expensive. VMA can also be used for monitoring therapy. Molecular defects of prognostic value in neuroblastoma include the MYCN oncogene over expression, present in about $25 \%$ of patients. Over expression of MYCN overexpression is a poor prognostic indicator (6). Elevation of serum ferritin, neurone specific enolase, (NSE), and lactate dehydrogenase, (LDH), have been associated with poor prognosis, and would be particularly useful in centres without facilities to undertake molecular studies. While LDH and serum ferritin are useful for disease monitoring response to treatment, NSE maybe useful in the differentiation of stage IVs from Stage IV, as the latter is associated with much higher levels (7).

Disease stage and age at presentation are the most important predictors of prognosis of neuroblastoma $(1,8)$. Indeed countries such as Japan, Canada, USAand United Kingdom have previously carried out VMA screening programmes in children below the age of one year, using highly sensitive chromatographic techniques, with the aim of detecting early, sub clinical neuroblastoma to improve prognosis. Although the value and cost effectiveness of these mass screening programmes remain controversial, this illustrates the prognostic importance placed on early detection of neuroblastoma (1). The International Neuroblastoma Staging System, (INSS), is one of the most widely used methods of staging neuroblastoma (6). Advanced disease particularly stage IV is associated with very poor prognosis while localised stages I, II, has very favorable prognosis (3).

In the developed world, about 55\% of neuroblastoma patients present with advanced poor prognosis stage VI disease. Infants aged one year and below, have a very good prognosis with cure rates as high as $80 \%$ reported, even with advanced Stage IVS disease $(1,8)$.

Currently patients diagnosed with neuroblastoma in developed countries are stratified into three major risk groups; the low risk category, intermediate risk and a high risk category. Patients with localized Stages I and II disease, favorable histology, and without MYCN amplification are 
classified as low risk. Infants below one year are also included in the low low risk category including those with Stage VIS disease. Treatment of this low risk group includes radical surgical excision of tumour, low intensity chemotherapy and radiotherapy in some patients (1). Criteria for high risk assignment include advanced stage IV disease, presence of MYCN gene amplification and age above one year. These high risk patients are treated with high intensity and myelosuppressive therapy which may necessitate bone marrow rescue with haemopoietic cell transplants. Intermediate risk patients may have some features of low risk and high risk categories, and are treated with intermediate intensity chemotherapy generally. Novel therapies include targeted radiotherapy and immunotherapy. This risk adapted treatment approach of neuroblastoma which matches clinical -pathologic features with treatment modality has yielded very significant improvement in survivals of neuroblastoma patients in the developed world. Event free survival of up to $90 \%$ has been reported in the low risk groups. Up to $40-60 \%$ long term survivals in the intermediate and low risk groups (6) are being achieved. These superior treatments outcomes are a result of continuous reviews and updates of treatments which are carried out every two years or so. Such frequent reviews maybe unaffordable in resource limited set ups. However efforts should nevertheless be made to carry out the reviews about every five years.

In Kenya, the last review on neuroblastoma treatment outcomes, but which did not even include clinical-pathologic features, was carried out by Kasili et al (9) over 20 years ago. The present study was carried out to determine both clinical-pathologic characteristics and treatment outcomes in children admitted for neuroblastoma treatment at $\mathrm{KNH}$. The findings of this study may be useful in renewing attention to presentation and management status of this childhood malignancy whose local importance is often overshadowed by the more prevalent childhood malignancies like lymphomas, nephroblastoma and leukemia's.

\section{MATERIALS AND METHODS}

Study design, setting and subjects: This was a crosssectional descriptive study based on review of patients' records. The study period was January 1997 to December 2005. Records of all children aged 15 years and below, with the diagnosis of neuroblastoma, were retrieved and data extracted using pre-designed data extraction sheets.

Case definition: Only subjects with a histopathology or cytology, (tumour, bone marrow or both), diagnosis consistent with neuroblastoma were eligible for inclusion. Cases with clinical features of neuroblastoma, but doubtful histology or cytology, were included if the urinary VMA was positive.

Data collection: All records of eligible patients were retrieved and scrutinised. Information on age at presentation, sex, presenting complaint(s) and duration, which included presence of swelling and their sites, pain and inability to walk, evidence of neurological disease, non specific signs and symptoms, (such as weakness, fever, diarrhoea, vomiting, irritability, and excessive sweating) were captured and recorded in pre-designed data extraction sheets. Laboratory data including full blood counts, clinical chemistry, (liver function and kidney function tests, LDH, ferritin, uric acid and urinary VMA screening results, histopathology reports, FNA and bone marrow cytology reports were also recorded. Diagnostic imaging results, (chest X-rays, skeletal surveys, abdominal ultrasound, CT scans and MRI reports), were also recorded. Staging of disease was noted and recorded, and where not indicated retrospective staging done according to the INSS. Treatment and treatment modalities were included. For patients who had surgery, the type and reason for surgery was also extracted. Where chemotherapy was given, the protocol, number of courses given and completed was recorded. Response to treatment was classified as complete, partial, minor or none. Patients who got radiotherapy and their response to radiotherapy were noted. Survival was measured from the time the patient was first seen at $\mathrm{KNH}$, to the last time they were seen alive with or without disease. Where patients were lost to follow up with progressive disease, or transferred to the nearest hospital with unresponsive disease, this was also noted.

Dataanalysis: The data was computerised cleaned and verified using theSPSS version 10.0 software. Analysis was basically frequency analysis, giving rise to median and descriptive proportions. Baseline characteristics were tabulated and frequency of variables calculated. Ninety five per cent Confidence intervals were calculated for primary outcome measures to indicate accuracy of the estimates. P-values of less than 0.05 were considered as significant. Overall survival was calculated from the date of admission to the date of last follow up when status, (dead or alive) with presence or absence of malignant disease also noted.

\section{RESULTS}

Although 57 patient's files had a diagnosis of neuroblastoma, 31 cases were excluded from analysis because either the diagnosis was equivocal or the records were incomplete, leaving 26 cases. Age range was 5 days to 12 years, the median age five years. As shown in Table 1,50\% of the patients 
were aged two years and below. Male: Female ratio was 1:1, with 13 males and 13 females. Table 2 summarises the presenting features and shows that the commonest presenting features were abdominal swelling $14,(53.8 \%)$, inability to walk due to bone pains, $13,(50 \%)$, and head or orbital swellings, 10, (38.5\%). Over half of the patients had non specific symptoms, such as, fever, vomiting, irritability, diarhoea and fatigue. Based on clinical, laboratory and diagnostic imaging, the abdomen $(88.9 \%)$; skeleton $(84.6 \%)$, bone marrow $(75 \%)$ and the head and neck (36.8\%) were the most frequently involved sites as seen in Figure 1. In Table 3 it is noted that most patients, $24,(92.3 \%)$ presented with advanced stage IV disease. Table 4 shows laboratory characteristics at presentation. Anaemia was common with $16,(72.7 \%)$ patients having an $\mathrm{Hb}$ equal to or less than $8 \mathrm{~g} / \mathrm{dl}$. Bone marrow involvement was present in 12 out of 16 $(75 \%)$ patients examined. One patient presented with an isolated testicular mass, whose diagnosis was confirmed with immunohistochemistry.

Table 1

Distribution of neuroblastoma patients at presentation by age and gender $(n=22)$

\begin{tabular}{lccccc}
\hline Age (years) & Male & Female & Total & $(\%)$ & Cumulative $(\%)$ \\
\hline$>1$ & 4 & 3 & 7 & 31.8 & 31.8 \\
$1-2$ & 3 & 1 & 4 & 18.2 & 50 \\
$3-6$ & 0 & 3 & 3 & 13.6 & 63.6 \\
$7-10$ & 1 & 3 & 4 & 18.2 & 81.5 \\
$11-15$ & 3 & 1 & 4 & 18.2 & 100 \\
\hline Total & 11 & 11 & 22 & 100 & \\
\hline
\end{tabular}

NB: The age was not clearly indicated in the records of four patients

Table 2

Presenting clinical features in 26 children with neuroblastoma

\begin{tabular}{lcc}
\hline Clinical feature & No. of patients & $(\%)$ of total \\
\hline Abdominal swelling & 14 & 53.8 \\
Inability to walk and bone pains & 13 & 50 \\
Swellings on the head and proptosis & 10 & 38.5 \\
Fever & 5 & 19.8 \\
Weakness & 4 & 15.4 \\
Vomiting & 3 & 11.5 \\
Sweating & 2 & 7.7 \\
Swellings elsewhere (testis and femur) & 2 & 7.7 \\
Others (one each)* & 3 & 11.5 \\
\hline
\end{tabular}

${ }^{*}$ Diarrhoea, convulsions and superior vena caval syndrome

Note: Some patients had more than one presenting symptoms 
Table 3

Disease stage at presentation in 26 children with neuroblastoma according to the International Neuroblastoma Staging System

\begin{tabular}{|c|c|c|c|}
\hline Stage & No. of patients & $\%$ of patients & Staging criteria \\
\hline I & 0 & 0 & $\begin{array}{l}\text { Localised tumour with complete gross excision, } \\
\text { with or without microscopic residual disease: } \\
\text { representative ipsilateral lymph nodes negative for } \\
\text { tumour microscopically, (nodes attached to and } \\
\text { removed with the tumour maybe positive) }\end{array}$ \\
\hline IIA & 0 & 0 & $\begin{array}{l}\text { Localised tumour with or without complete gross } \\
\text { excision with ipsilateral non-adherent lymph nodes } \\
\text { negative for tumour. Enlarged contra-lateral lymph } \\
\text { nodes must be negative tumour microscopically }\end{array}$ \\
\hline IIB & 1 & 3.8 & $\begin{array}{l}\text { Localised tumour with or without complete excision, } \\
\text { with ipsilateral nonadherent lymph nodes positive } \\
\text { for tumour. Enlarged Contra- lateral lymph nodes } \\
\text { must be negative microscopically }\end{array}$ \\
\hline III & 0 & 0 & $\begin{array}{l}\text { Unresectable unilateral tumour infiltrating across } \\
\text { the midline, with or without regional lymph node } \\
\text { involvement; or localised unilateral tumour with } \\
\text { contra- lateral regional lymph node involvement: or } \\
\text { midline tumour with bilateral extension by infiltration } \\
\text { (unresectable) or by lymph node involvement }\end{array}$ \\
\hline IV & 24 & 92.3 & $\begin{array}{l}\text { Any primary tumour with dissemination to distant } \\
\text { lymph nodes, bone, bone marrow, liver, skin or } \\
\text { other organs (except Stage } 4 S^{*} \text { ) }\end{array}$ \\
\hline IVS & 1 & 3.8 & $\begin{array}{l}\text { Localised primary tumour as defined for stages } 1,2 \mathrm{~A} \\
\text { or } 2 \mathrm{~B} \text {, with dissemination limited to skin, liver, and } \\
\text { or bone marrow involvement ( } 10 \% \text { involvement) }\end{array}$ \\
\hline
\end{tabular}

Table 4

Laboratory characteristics of children with neuroblastoma before commencement of treatment

\begin{tabular}{lll}
\hline Characteristic & No. & $(\%)$ \\
\hline $\mathrm{Hb}^{*}$ level (g/dl) & & \\
$8.0-5.0$ & 16 & 72.7 \\
Bone marrow cytology done & 16 & 72.7 \\
Bone marrow involvement among those & 12 & 75.0 \\
Who had bone marrow aspiration & & \\
FNA-C ${ }^{* *}$ of tumour done & 18 & 81.8 \\
Positive for neuroblastoma & 16 & 72.7 \\
Tissue biopsy for diagnosis (n= 16) & 8 & 50 \\
Number who had histological grading & 2 & 12.5 \\
Number who had immunohistochemistry & 2 & 12.5 \\
Urinary screening for VMA ** & 12 & 54.5 \\
Number with positive VMA & 6 & 50.0 (of those tested) \\
Number whose LDH level was tested & 3 & 13.6 \\
Number with elevated LDH & 3 & 100 (of those tested) \\
\hline
\end{tabular}

${ }^{*} \mathrm{Hb}=$ Haemoglobin. The lowest $\mathrm{Hb}$ was $5.4 \mathrm{~g} / \mathrm{dl}$

${ }^{* *}$ FNAC $=$ Fine needle aspirate cytology

${ }^{* * *}$ VMA = Vanilyl mandelic acid 
Figure 1

Frequency of involvement of various body sites at presentation

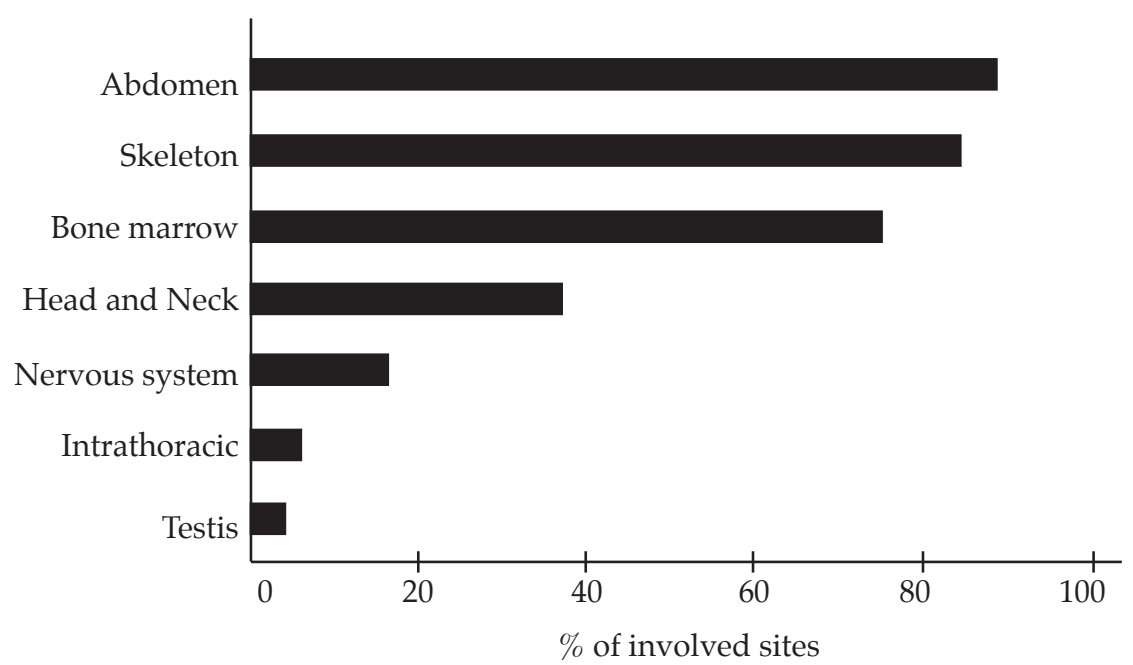

Table 5

Results of imaging in children with neuroblastoma, (before treatment)

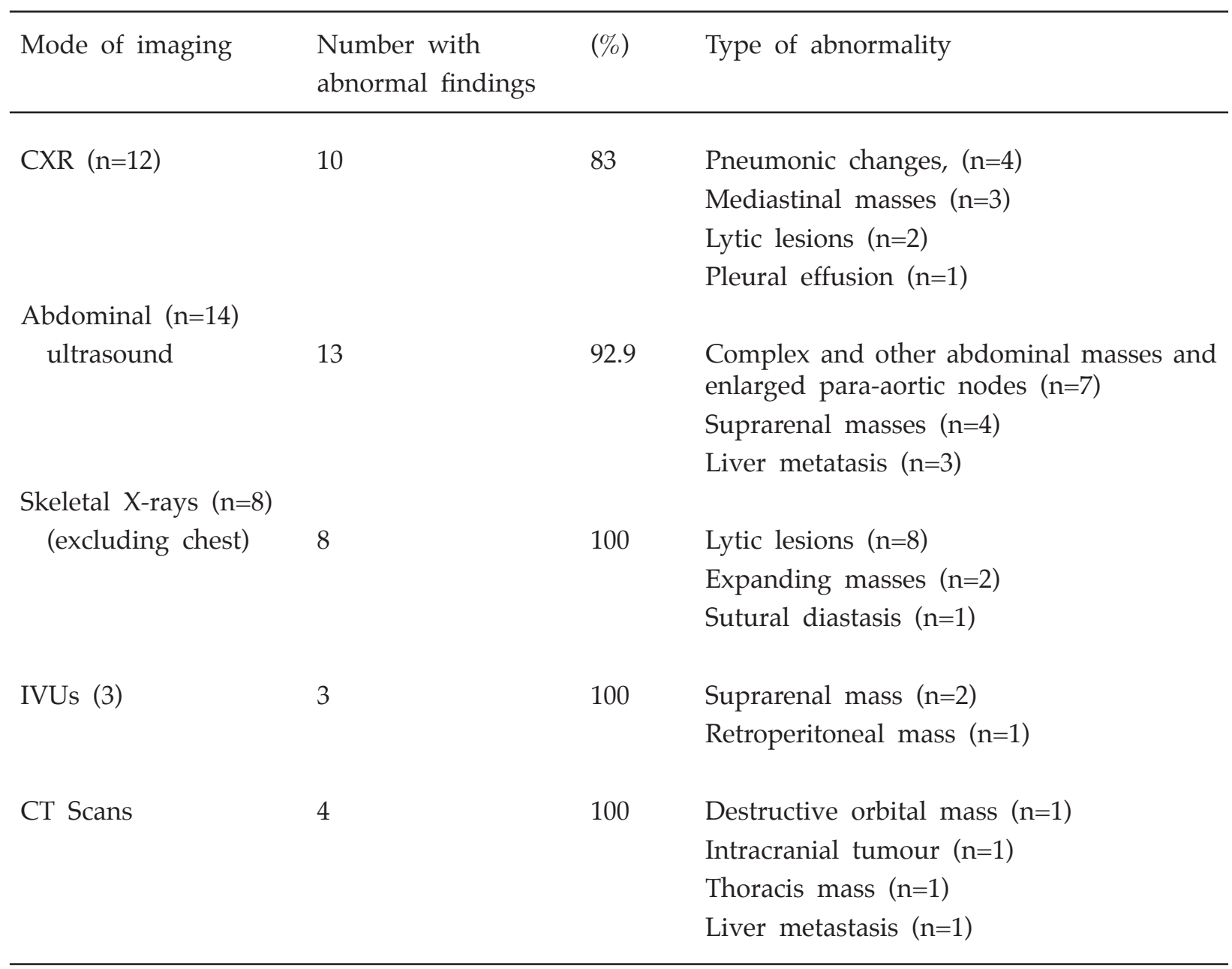

NB: Some patients had more than one type of imaging, while some had multiple lesions 
Urinary VMA screening using visual colour change method, was positive in 50\% (CI 29.9- 70.1\%), of the patients who were screened. LDH was elevated in all the four patients for whom it was done, (at diagnosis) and results were available. None of the patients had serum ferritin or NSE levels estimated. Liver transaminases were elevated in two patients, one of whom had a positive hepatitis $B$ surface antigen. Renal functions were normal in all patients. Histopathology was the basis for diagnosis in 50\% (CI 40.6-79.80\%) patients' records available, while the rest had a diagnosis based on FNA of tumour or bone marrow cytology together with VMA findings. Two patients, $(8 \%)$, who had difficult histology, had confirmation of diagnosis by immunohistochemistry done in a foreign country. These two histologies also had histological grading indicated on the reports carried out overseas. Amongst the 12 patients who had FNAcytology, two also had tissue biopsy and in these cases cytology and histopathology results concurred. Table 5 summarises the types and results of the patient's imaging. Abnormal imaging results were present in majority of the patients.

\section{Table 6}

Treatment outcome and survival of neuroblastoma patients

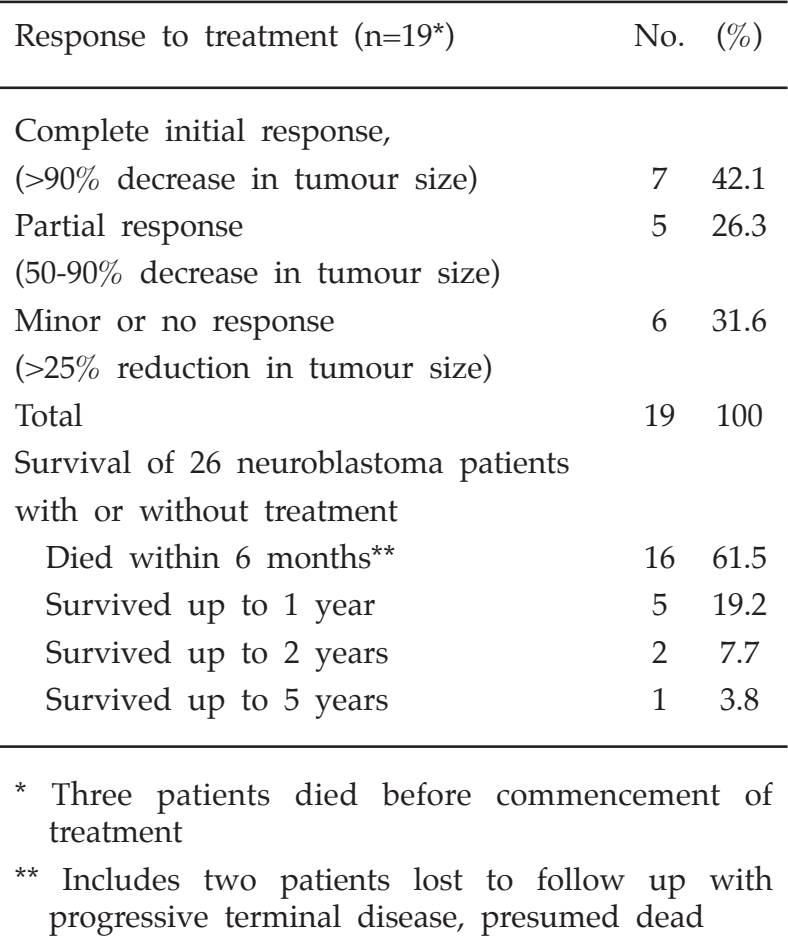

Table 6 shows treatment response and survival of the patients. Three very ill patients died before treatment commencement. The rest of the patients all $(100 \%)$ underwent combination chemotherapy with vincristine, cyclosphosfamide, adriamycin and cis- platin by either one of 2 protocols $(10,11)$. Radiotherapy was given to only one patient who had a complex pelvic mass compressing the ureters and causing hydronephrosis, a hydrocoele and renal failure. The tumour responded favorably to radiotherapy initially with partial regression of the tumour. However recurrence at the same site within a few weeks, led to death of the patient from renal failure.

Majority of the patients had initial complete or partial response to chemotherapy. However most had subsequent relapse and resistance to chemotherapy and either died or were discharged with progressive disease. Overall survival at one and two years was 19.2\% (CI 6.6- 39.4\%) and 7.7\% (CI $0.925-25.1 \%$ ) respectively. Only one patient, $(3.8 \%)$, who had presented with stage 2B disease, survived, (also disease free) beyond five years (3.8\%).

\section{DISCUSSION}

The clinical presentation of patients in this study are very similar to what has been described elsewhere in both developed and developing countries (3, 6, 12). The youngest patient presented at $\mathrm{KNH}$ with anaemia and abdominal distension at the age of five days. According to the history, abdominal masses and anaemia were noted soon after birth. Most probably this patient had congenital neuroblastoma. Half of the patients were aged two years and below. The commonest presenting features included swellings of the abdomen, inability to walk due to bone pains and non specific symptoms such as weakness, fever, vomiting and sweating. The most frequently involved sites were the abdomen, skeleton, head and neck. Bone marrow involvement and anaemia were frequent. These findings are comparable with what is reported in the literature $(1,3,13)$.

However majority presented with advanced stage IV disease. One unusual case was a patient who presented with an isolated testicular swelling, a rare finding in neuroblastoma (1). Despite complete excision of the tumour, followed by chemotherapy and radiotherapy, there was aggressive tumour regrowth with compression of the ureters, renal failure and death of the patient.

Ideally all patients with neuroblastoma should have a tissue diagnosis in which the risk category is indicated to guide in the decision of appropriate intensity of treatment (1). Half of the patients whose data was available did not have tissue diagnosis. To 
improve our treatment outcomes of neuroblastoma, it will be necessary to ensure that all patients suspected to have neuroblastoma have tissue biopsy, not only to improve the accuracy of diagnosis, but to also help with risk categorisation and possible risk adaptation of the treatment.

Only half of patients whose records could be evaluated had VMAscreening. Expensive techniques like immunohistochemistry may not be affordable in public hospitals in Kenya but it is recommended that more affordable tests like a sensitive VMA test and serum ferritin be made readily available to improve on the diagnosis of neuroblastoma in resource constrained set ups.

Although surgery and radiotherapy have important roles in treatment of neuroblastoma (12), only one patient had surgery as a debulking procedure in this study. This patient had the best prognosis. Radiotherapy was given to only one patient as a palliative measure. The limited role of surgery and radiotherapy is most likely related to the late presentation of the patients in this study.

Over 20 years back, Kasili et al (9) noted that neuroblastoma had the poorest prognosis of all paediatric solid tumours managed at the KNH. Healso noted that this was associated with late presentation of patients. The prognosis of neuroblastoma remains very poor compared to the greatly improved results recorded by developed countries $(1,3,9)$. As long as patients continue to present with advanced disease, the prognosis of neuroblastoma is likely to remain poor irrespective of the quality of care or any other interventions.

Asignificant limitation of this study was the small number of subjects that met eligibility criteria because of missing data. This is however not surprising with a retrospective study. A prospective study is therefore recommended which should avail more precise information.

\section{REFERENCES}

1. Orkin, S.H., Fisher, D.F., Look, A.T. et al. Oncology of Infancy and Childhood. $1^{\text {st }}$ Edition, 2009: Saunders Elservier. Philadelphia PA, USA.

2. Macharia, W. M. Childhood cancers in a refferal hospital in Kenya, a review. East Afr. Med. J. 1996; 73: 647-650.

3. Lucayo,J.N., Mariner, N. andDavis, L. Neuroblastoma emedicine. Updated July 2009. Last accessed $4^{\text {th }}$ December 2009.

4. Kalebi, A.Y. and Dada, M. A. Application of immunohistochemistry in clinical practice: A review. East Afr. Med. J. 2007; 84: 389-397.

5. La Brosse, E.H. Urinary excretion of 3-methodoxy hydroxymandelic acid and 3-methodroxyphenyl aceti acid. Cancer. 1980; 40: 1995-2001.

6. Imbach, P. and Kuhne, I. Arceci in Pediatric Oncology. Acomprehensive Guide. $2^{\text {nd }}$ Ed. 2006.Springer-Velag Berlin Heidelberg, Germany.

7. Berthold, F., Trechnow, R., Utsch, S., et al. Prognostic factors in metastatic neuroblastoma: a multivariate analysis of 182 cases. Am. J. Pediatr. Hematol. Oncol. 1992; 14: 2007-2015.

8. Stephen, S. R., et al. The prognostic significance of age and pattern of metastases in stage IVS neuroblastoma. Cancer. 1986; 58: 372-376.

9. Kasili, E. G., Kyambi, J. M. and Onyango, J. N. Treatment of childhood malignancies in Kenya. East Afr. Med. J. 1984; 61: 663-674.

10. Mwanda, O. W. Kasili's synopsis of management of childhood cancers in Kenya, page 96-97. Undated guidelines for management of childhood cancers at the KNH.

11. Macharia, W. M. Guidelines for management of paediatric malignancies in KNH. Undated alternative guidelines for the management of paediatric malignancies at the $\mathrm{KNH}$.

12. Evans, A.E. and D' Angio, J.G. The role of multimodal therapy in patients with local and regional neuroblastoma. J. Paed. Surg. 1984; 19: 77-80.

13. Familusi, J. B., Aderere, W. I. and Williams, O. A. Features of neuroblastoma in African children. East Afr. Med. J. 1978; 55: 182-190. 